\title{
CARACTERIZACIÓN Y PROPUESTA DEL PLAN DE DESARROLLO URBANO DE LA CIUDAD DE MAÑAZO
}

\author{
CARACTERIZACIÓN Y PROPUESTA DEL PLAN DE DESARROLLO \\ URBANO DE LA CIUDAD DE MAÑAZO
}

\author{
${ }^{1}$ Ingrid Chaiña Flores
}

\begin{abstract}
RESUMEN
El propósito de esta investigación es conocer las características físicas, espaciales, ambientales y estilos arquitectónicos de las edificaciones de la ciudad de Mañazo para proponer el plan de desarrollo urbano, que viabilice una ciudad sostenible. Este centro urbano presenta un proceso de crecimiento de manera espontánea e irregular, generando asentamientos humanos informales, que están destruyendo progresivamente singular paisaje natural, privándola de sus principales atractivos y potencialidades. Entre los principales resultados se tiene: En el aspecto físico espacial, este centro urbano es carente de una visón de desarrollo integral, existe la necesidad urgente de establecer lineamientos estratégicos para su desarrollo urbano, desde la ampliación de la infraestructura de servicios básicos, hasta los aspectos económicos, sociales y ambientales; cuya resultante sea una ciudad que brinde a su población condiciones favorables para vivir en los tiempos actuales, con oportunidades para los diferentes grupos poblacionales, principalmente en actividades productivas y pos producción, aprovechando el potencial turístico y dotarle sentido de vida.
\end{abstract}

Palabras clave: Características físicas, desarrollo urbano, espacio, sentido de vida.

\section{ABSTRACT}

The purpose of this research is to understand the physical, spatial, environmental characteristics and architectural styles of the buildings of the city of Mañazo to propose the plan of urban development, which guarantee a sustainable city. This urban center appears a process of growth in a spontaneous and irregular, generating informal human settlements, which are destroying progressively unique natural landscape, depriving it of its main attractions and potential. Among the main results are: spatial appearance, this urban center is devoid of a comprehensive development vision, there is urgent need to establish strategic guidelines for its urban development, since the expansion of the infrastructure of basic services, economic, social and environmental aspects; whose result is a city that provides its people favorable conditions to live in modern times, with opportunities for the different population groups, mainly in productive activities and post production, taking advantage of the tourism potential and provide a sense of life

Keywords: physical characteristics, urban development, space, sense of life.

\section{INTRODUCCIÓN}

El propósito de este estudio es caracterizar y proponer el plan de desarrollo urbano en base a las particularidades de los aspectos sociales, económicos, ecológicos y ambientales de la capital del distrito de Mañazo. Este contexto particular no se encuentra aislado del desarrollo global de la sociedad, sino inmerso en la realidad provincial, regional, nacional y global del sistema mundo; por lo que, es necesario visualizar la particularidad distrital dentro de este marco global.

La apertura económica y comercial del país hacia un mundo global, ha generado importantes actividades productivas, a ello se suma la creación de gobiernos locales y regionales con la capacidad de coadyuvar al desarrollo integral de espacios locales. Las perspectivas de satisfacer las distintas necesidades de la población de esta ciudad, con nuevas propuestas que constituyen el punto de partida para dar respuesta a las exigencias del crecimiento urbano con enfoque de desarrollo sostenible y armónico.

\section{MATERIALES Y MÉTODOS}

El ámbito de estudio se encuentra ubicado a $44 \mathrm{~km}$. al noreste de la ciudad de Puno; geográficamente se ubica a $15^{\circ}$ $47^{\prime} 54^{\prime \prime}$ de Latitud Sur y a $70^{\circ} 20^{\prime} 28^{\prime}$ ' de Longitud Oeste, a una altura promedio de 3926 m.s.n.m. La zona urbana cuenta con una extensión aproximada de 300 hectáreas. Como referencia, “... el altiplano de Puno se encuentra entre 3,700 y 4800 m.s.n.m.” (Gutiérrez, 2015).

\footnotetext{
'1 Arquitecta. Docente de la Escuela Profesional de Arquitectura y Urbanismo de la Facultad de Ingeniería Civil y Arquitectura de la Universidad Nacional del Altiplano. Puno Perú
} 
Chaiña, I. Caracterización y propuesta del plan de desarrollo urbano de la ciudad de Marazo.

En función al propósito, el tipo de investigación es aplicado, porque direcciona hacia el diseño e implementación de un plan de desarrollo urbano. En lo referente al nivel de análisis es descriptivo, porque diagnostica y desarrolla la propuesta.

El eje temático está contenida en la planificación urbana, constituida por el sujeto y el objeto, en el primer eje, se considera la población del centro urbano y el sistema de actividades que se desarrolla y en el segundo eje, la ciudad en sus aspectos físicos y ambientales que sustentan las actividades del sujeto en este espacio.

La investigación tiene un enfoque predominantemente espacial y se basa en estudios físicos y ambientales. Esta etapa hace posible analizar los componentes de la ciudad y proponer los principios que, apoyados con el análisis de aptitud integral y en la misión como imagen actual y visión compartida de futuro, permiten definir las propuestas del Plan de Desarrollo Urbano de la ciudad de Mañazo. En este enfoque las propuestas constituyen, la síntesis de las políti- cas de desarrollo de este centro urbano y ellas quedan expresadas en los usos de suelo, el plan vial y transportes y el plan de expansión urbana, en perspectiva futura.

\section{RESULTADOS Y DISCUSIÓN}

Las características de la situación problemática de la ciudad de Mañazo, surgen de la necesidad de un planeamiento adecuado, en respuesta a la situación actual de la ciudad referente a su sistema físico, ambiental, vial y transporte, social, económico, entre otros. Este centro urbano por su ubicación, paisaje, actividades económicas, carece de un protagonismo dentro de la provincia y región, por la falta de promoción, difusión y políticas de desarrollo urbano con enfoque sostenible. Actualmente esta ciudad no cuenta con una infraestructura de equipamiento urbano adecuado a su realidad y demandas.

En el marco de esta situación problemática, se tiene

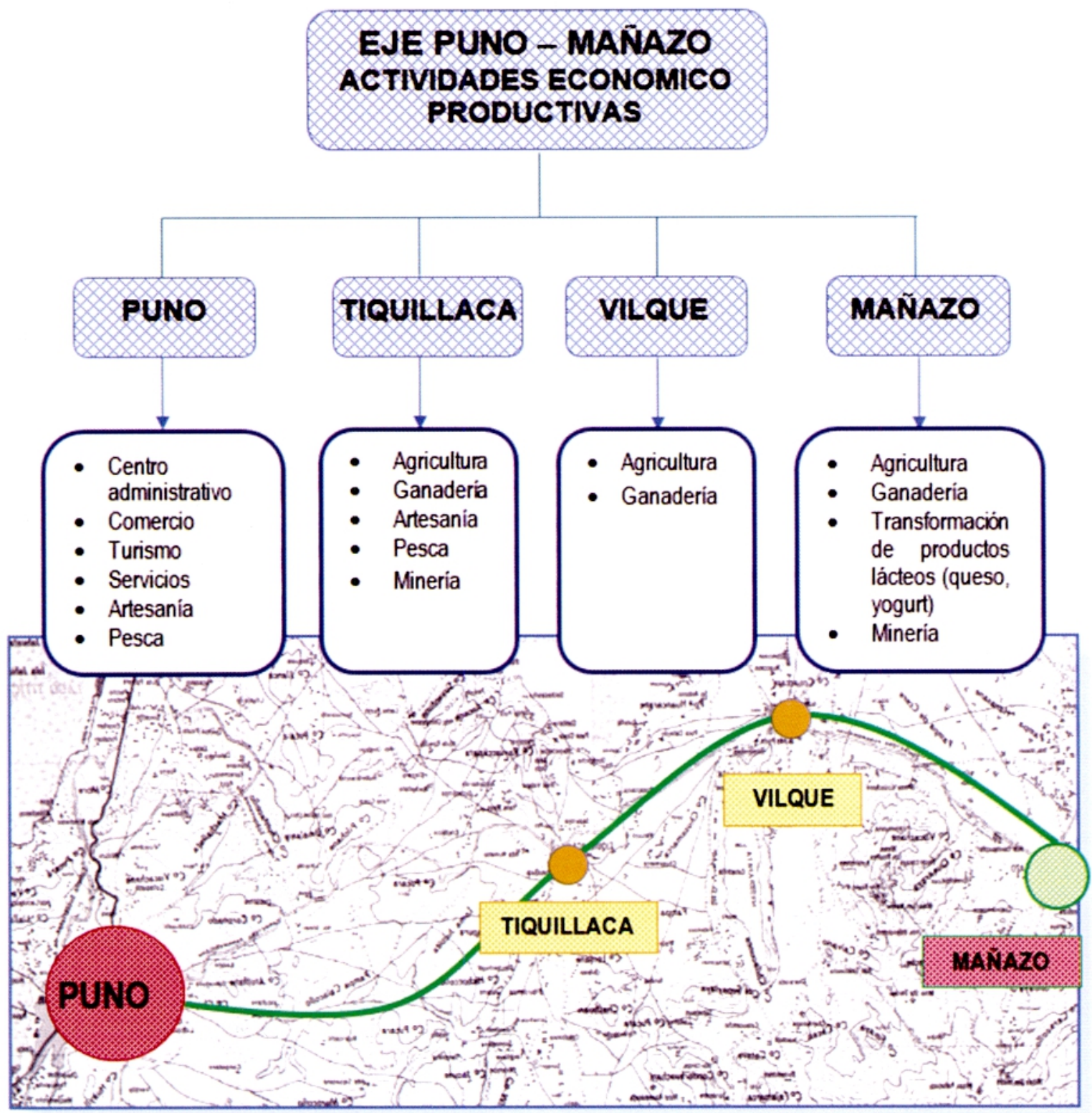

Figura 1. Actividades económico-productivas. Fuente: Elaboración propia 
Chaiña, I. Caracterización y propuesta del plan de desarrollo urbano de la ciudad de Marazo

un gran desafio, construir un centro urbano con perspectiva social, económica y ambiental; pero a su vez que mejore la calidad de vida, de manera tal que la prosperidad de sus habitantes y que las generaciones futuras tengan las condiciones favorables para resolver retos mayores. El contexto urbano está estrechamente relacionado con las economías familiares rurales; al respecto Mayer (2004) considera tres elementos -el hogar, los campos y el dinero- “... El hogar, y la casa que lo cobija, son el lugar de refugio, almacenaje, crecimiento, identidad y autonomía. Los campos también forman parte de la unidad doméstica; son el lugar donde las semillas se convierten en cosechas. Y el dinero comprende aquellas fichas que los miembros de la familia luchan sin cesar por obtener y aman gastar en bienes de consumo, como bebidas, música y ropas".

La definición de escenarios, obedece a la necesidad de identificar las diferentes opciones de desarrollo que tendría a futuro la ciudad de Mañazo en horizontes de corto, mediano y largo plazo, opciones que parten de un análisis de las tendencias favorables o desfavorables de las variables claras provenientes de la caracterización. El escenario tendencial, refleja el futuro de la ciudad teniendo en cuenta la evolución actual, bajo el supuesto que no se producirán intervenciones de importancia por parte de los diferentes actores locales. El escenario probable, plantea el futuro de la ciudad, bajo el supuesto de que se producirán ciertas intervenciones aisladas por parte de los agentes locales, regionales y nacionales; $y$ el escenario deseable, prevé el futuro de la ciudad bajo el supuesto de que se concentrarán las principales estrategias del plan de desarrollo urbano en forma sistemática, oportuna e interrelacionada, en perspectiva de la consecución de la visión como imagen futura.

Las características de la situación contextual y los escenarios de la ciudad de Mañazo, direccionan diseñar algunos componentes estratégicos a saber: Ordenamiento territorial, promoción del crecimiento urbano, ordenamiento urbano paisajístico, estructuración del sistema vial urbano y de transportes, modernización de la gestión urbana ambiental, y promoción de la equidad social urbana.

La implementación del modelo de ordenamiento territorial sostenible del distrito de Mañazo, requiere la consolidación y desarrollo de infraestructura económica, la optimización de articulación vial distrital con la red regional y nacional, la dotación y mejoramiento de infraestructura de servicios básicos y equipamiento social, y de la implementación y monitoreo de planes urbanos y catastros municipales.

La promoción del crecimiento urbano competitivo, requiere la consolidación de áreas urbanas productivas; el reordenamiento y puesta en valor de Mañazo tradicional; la implementación de servicios comerciales y promoción de servicios turísticos, culturales y eco-recreativos. El ordenamiento urbano y paisajístico, requiere la promoción de sistema de centro y sub centros de servicios, tratamiento urbanístico paisajístico de la ciudad, y aplicación de reglamentación de uso de suelos.

La estructuración del sistema vial urbano y de transportes, requiere de la implementación de propuesta del sistema vial urbano, aplicación de reglamentación vial y de transportes, y reordenamiento de transporte terrestre. La modernización de la gestión urbana ambiental, necesita de la institucionalización de planes de desarrollo local, establecimiento de agenda y mecanismos de concertación de acciones de desarrollo urbano sostenible, y fortalecimiento y consolidación de mecanismos de participación ciudadana.

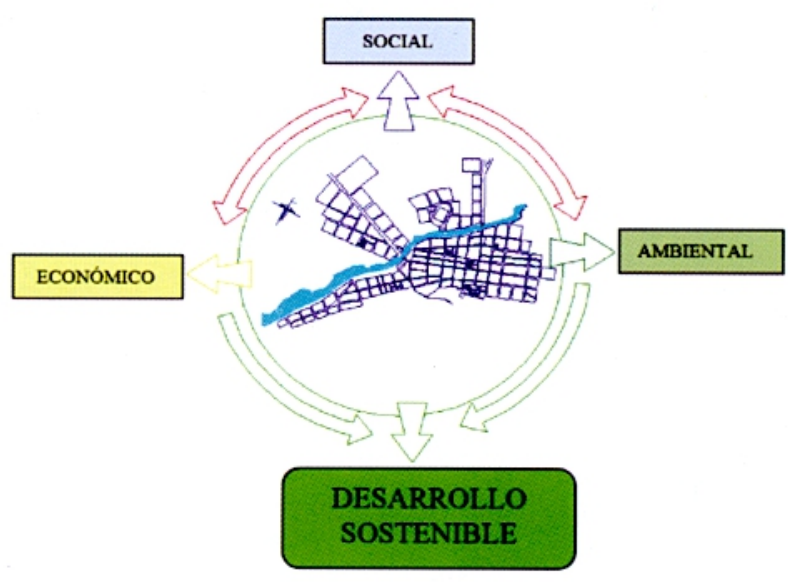

Figura 2. Concepción de ciudad sostenible Fuente: Rogers. Richard, 1997. Ciudades para un Pequeño Planeta

La promoción de la equidad social urbana, requiere del establecimiento de programas de promoción de empleo urbano, apoyo de programas de lucha contra la pobreza, y del establecimiento de programas de educación ciudadana y de cultura urbana. Al respecto (Vicentelo, P. 2003) al referirse al desarrollo humano y las principales concepciones contemporáneas de desarrollo, considera: Las teorías sobre desarrollo han evolucionado desde una corriente economicista de desarrollo, hasta una corriente que presta atención prioritaria al ser humano y su entorno.

El instrumento técnico normativo del desarrollo sostenible, será el plan de desarrollo urbano que buscará consolidar a la ciudad de Mañazo como un centro estructurado y en armonía con su territorio, para ello se fortalecerán los elementos estructurantes urbano-naturales, como los ríos y las quebradas como corredores naturales recuperadas de la contaminación y como elementos ambientales con relieve con una amplia oferta paisajista y recreativa. "El medio ambiente es el espacio en el que se desarrollan las actividades humanas" (Vera, 2014).

Una ciudad con calidad ambiental, sostenible, en armonía con el medio natural, que refuerce la identidad y la cohesión social, la autoestima y el orgullo por su ciudad de todos los sectores de la población. Así mismo, teniendo en cuenta las características del territorio y el conjunto de espacios que conforma este ámbito de intervenciones; las áreas urbanas y áreas pre-urbanas integradas al centro urbano, la cual determina unidades de desarrollo; sumado a la dinámica que genera el ámbito regional; impulsando en lo específico el sector primario y terciario, y dentro de este, al subsector comercial y a la recreación y deporte como actividades complementarias.

\section{CONCLUSIONES}

En lo físico espacial, el centro urbano de Mañazo requiere una visión de desarrollo integral que establezca los 


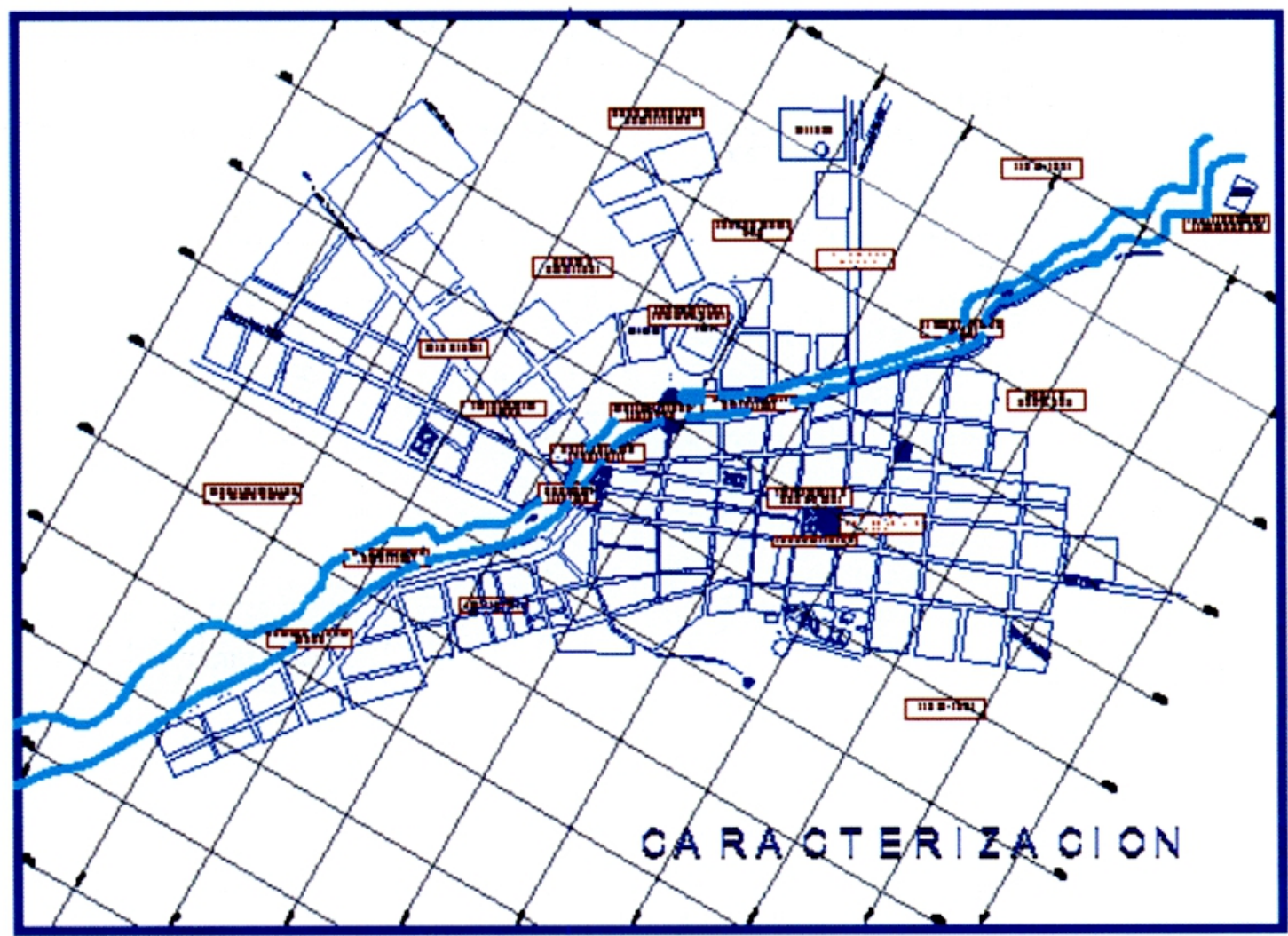

Figura 2. Plano de caracterización de la ciudad de Mañazo Fuente: Elaboración propia

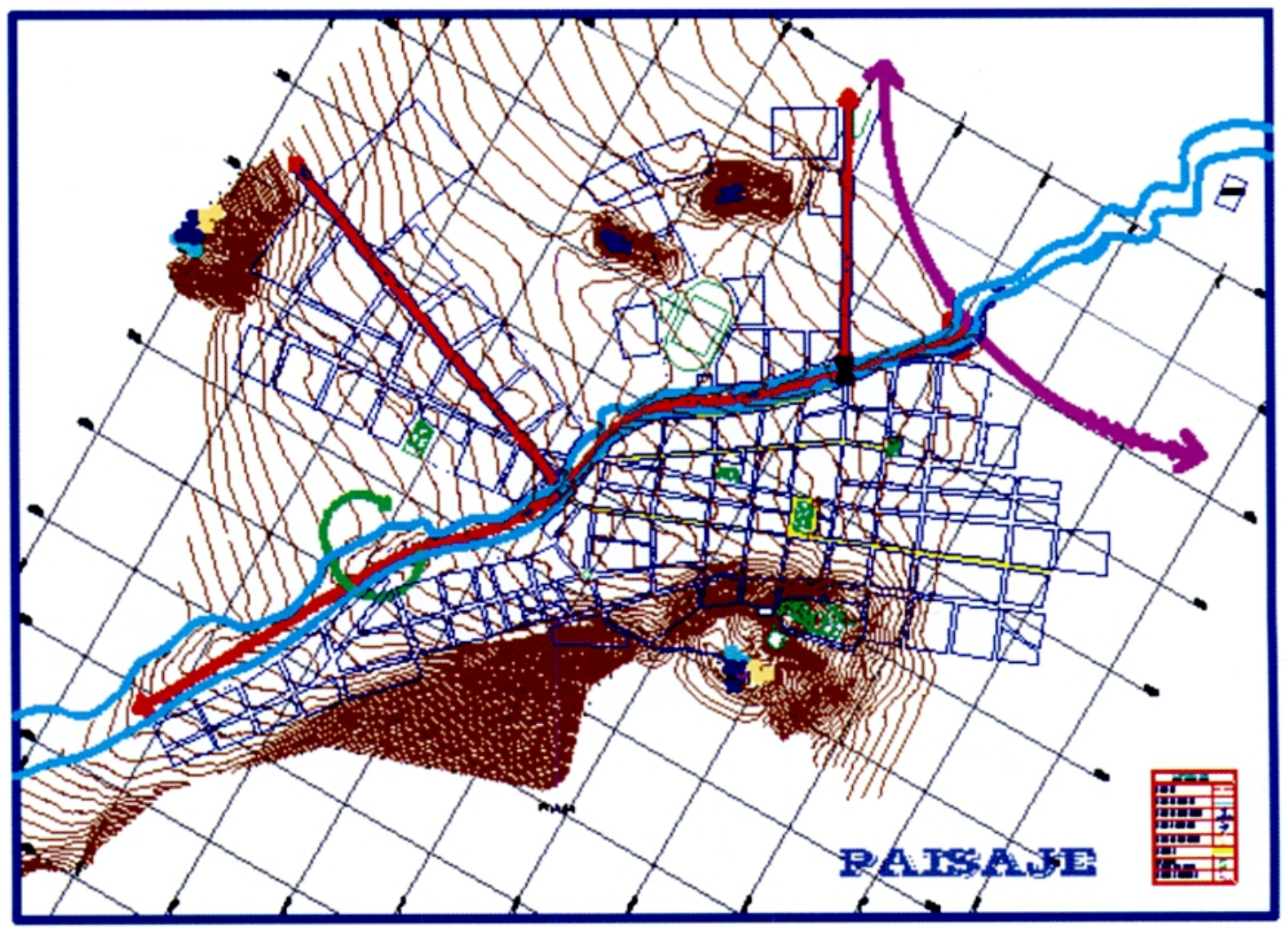

Figura 3. Plano de paisaje urbano y contexto espacial Futente: Elaboración propia. 
Chaiña, I. Caracterización y propuesta del plan de desarrollo urbano de la ciudad de Marazo.

lineamientos estratégicos para su desarrollo urbano, tiene la necesidad urgente de la ampliación de infraestructura de sus servicios básicos, y requiere del mejoramiento de su infraestructura vial, sin embargo cuenta con escasas vías pavimentadas a partir de la plaza principal.

En lo ambiental, es necesario la previsión de un sistema de tratamiento de residuos sólidos urbanos; la laguna de estabilización requiere mayor tratamiento, evitar que se muestre como zona de contaminación ambiental.

En lo social, la población del centro urbano de Mañazo está sufriendo un proceso de emigración a las ciudades importantes de la región y el país, por deficientes condiciones favorables para un desarrollo sostenible; la población joven del centro urbano prefiere salir a buscar nuevas oportunidades, por ello la ausencia de población joven y el no crecimiento poblacional.

En lo económico, la actividad del centro urbano es de gestión de la producción agropecuaria y de derivados lácteos que se realiza en pequeña escala, haciendo que esta sea en más del $50 \%$ una economía de auto subsistencia, debido a que se produce para el consumo familiar y para el mercado; el centro urbano presenta un potencial turístico, pero por el estado de las vías no son aprovechados adecuadamente.

El instrumento técnico normativo de gestión del desarrollo será el plan de desarrollo urbano que buscará consolidar a la ciudad de Mañazo como un centro urbano con calidad ambiental, sostenible, en armonía con el medio natural.

\section{REFERENCIAS BIBLIOGRÁFICAS}

Gutierrez, R. (2015). Arquitectura del altiplano perwano. Puno Perú: Altiplano E.I.R.L.

Mayer, E. (2004). Casa, Chacra y Dinero: Economías domésticas en los andes. Lima Perú: IEP.

Rogers, R. (1997). Ciudades para un pequeño planeta. Barcelona: Gill, S.A.

Vera, W. (2014). Arquitectura urbana de Puno. Puno Perú: Altiplano E.I.R.L.

Vicentelo, P. (2003). Indicadores de desarrollo bumano y su aplicación al campo agroecológico. Lima Perú: Escuela Para el Desarrollo.

\section{Correspondencia:}

Ingrid Chaiña Flores: nicollef21@hotmail.com
Fecha de Recepción: 20/10/2015

Fecha de Aceptación: 11/12/2015 